\title{
AURC from T1 to T2 Normalized by Surface Area
}

National Cancer Institute

\section{Source}

National Cancer Institute. AURC from T1 to T2 Normalized by Surface Area. NCI

Thesaurus. Code C92352.

The area under the urinary excretion rate curve (AURC) over the interval from T1 to T2 divided by the surface area. 